\title{
The $q$-Fractional Analogue for Gronwall-Type Inequality
}

\author{
Thabet Abdeljawad ${ }^{1,2}$ and Jehad O. Alzabut ${ }^{2}$ \\ ${ }^{1}$ Department of Mathematics and Computer Science, Çankaya University, Ögretmenler Caddesi 14, Balgat, 06530 Ankara, Turkey \\ ${ }^{2}$ Department of Mathematics and Physical Sciences, Prince Sultan University, P.O. Box 66833, Riyadh 11586, Saudi Arabia \\ Correspondence should be addressed to Jehad O. Alzabut; jalzabut@psu.edu.sa
}

Received 8 May 2013; Accepted 7 July 2013

Academic Editor: Dashan Fan

Copyright (c) 2013 T. Abdeljawad and J. O. Alzabut. This is an open access article distributed under the Creative Commons Attribution License, which permits unrestricted use, distribution, and reproduction in any medium, provided the original work is properly cited.

\begin{abstract}
We utilize $q$-fractional Caputo initial value problems of order $0<\alpha \leq 1$ to derive a $q$-analogue for Gronwall-type inequality. Some particular cases are derived where $q$-Mittag-Leffler functions and $q$-exponential type functions are used. An example is given to illustrate the validity of the derived inequality.
\end{abstract}

\section{Introduction}

The fractional differential equations have conspicuously received considerable attention in the last two decades. Many researchers have investigated these equations due to their significant applications in various fields of science and engineering such as in viscoelasticity, capacitor theory, electrical circuits, electroanalytical chemistry, neurology, diffusion, control theory, and statistics; see, for instance, the monographs [1-3]. The study of $q$-difference equations, on the other hand, has gained intensive interest in the last years. It has been shown that these types of equations have numerous applications in diverse fields and thus have evolved into multidisciplinary subjects [4-11]. For more details on $q$ calculus, we refer the reader to the reference [12]. The corresponding fractional difference equations, however, have been comparably less considered. Indeed, the notions of fractional calculus and $q$-calculus are tracked back to the works of Jackson [13], respectively. However, the idea of fractional difference equations is considered to be very recent; we suggest the new papers [14-28] whose authors have taken the lead to promote the theory of fractional difference equations.

The $q$-fractional difference equations which serve as a bridge between fractional difference equations and $q$ difference equations have become a main object of research in the last years. Recently, many papers have appeared which study the qualitative properties of solutions for $q$-fractional differential equations [29-33], whereas few results exist for $q$ fractional difference equations [34-36]. The integral inequalities which are considered as effective tools for studying solutions properties have been also under consideration. In particular, we are interested in Gronwall's inequality which has been a main target for many researchers. There are several versions for Gronwall's inequality in the literature; we list here those results which are concerned with fractional order equations [37-41]. To the best of authors' observation, however, the $q$-fractional analogue for Gronwall-type inequality has not been investigated yet.

A primary purpose of this paper is to utilize the $q$ fractional Caputo initial value problems of order $0<\alpha \leq 1$ to derive a $q$-analogue for Gronwall-type inequality. Some particular cases are derived where $q$-Mittag-Leffler functions and $q$-exponential type functions are used. An example is given to illustrate the validity of the derived inequality.

\section{Preliminary Assertions}

Before stating and proving our main results, we introduce some definitions and notations that will be used throughout the paper. For $0<q<1$, we define the time scale $\mathbb{T}_{q}$ as follows:

$$
\mathbb{T}_{q}=\left\{q^{n}: n \in \mathbb{Z}\right\} \cup\{0\},
$$


where $\mathbb{Z}$ is the set of integers. In general, if $\alpha$ is a nonnegative real number then we define the time scale

$$
\mathbb{T}_{q}^{\alpha}=\left\{q^{n+\alpha}: n \in \mathbb{Z}\right\} \cup\{0\},
$$

and thus we may write $\mathbb{T}_{q}^{0}=\mathbb{T}_{q}$. For a function $f: \mathbb{T}_{q} \rightarrow \mathbb{R}$, the nabla $q$-derivative of $f$ is given by

$$
\nabla_{q} f(t)=\frac{f(t)-f(q t)}{(1-q) t}, \quad t \in \mathbb{T}_{q}-\{0\} .
$$

The nabla $q$-integral of $f$ is given by

$$
\begin{gathered}
\int_{0}^{t} f(s) \nabla_{q} s=(1-q) t \sum_{i=0}^{\infty} q^{i} f\left(t q^{i}\right), \\
\int_{a}^{t} f(s) \nabla_{q} s=\int_{0}^{t} f(s) \nabla_{q} s-\int_{0}^{a} f(s) \nabla_{q} s, \quad \text { for } 0 \leq a \in T_{q} .
\end{gathered}
$$

The $q$-factorial function for $n \in \mathbb{N}$ is defined by

$$
(t-s)_{q}^{n}=\prod_{i=0}^{n-1}\left(t-q^{i} s\right)
$$

In case $\alpha$ is a nonpositive integer, the $q$-factorial function is defined by

$$
(t-s)_{q}^{\alpha}=t^{\alpha} \prod_{i=0}^{\infty} \frac{1-(s / t) q^{i}}{1-(s / t) q^{i+\alpha}}
$$

In the following lemma, we present some properties of $q$ factorial functions.

Lemma 1 (see [32]). For $\alpha, \gamma, \beta \in \mathbb{R}$, one has the following.

(I) $(t-s)_{q}^{\beta+\gamma}=(t-s)_{q}^{\beta}\left(t-q^{\beta} s\right)_{q}^{\gamma}$.

(II) $(a t-a s)_{q}^{\beta}=a^{\beta}(t-s)_{q}^{\beta}$.

(III) The nabla q-derivative of the q-factorial function with respect to $t$ is

$$
\nabla_{q}(t-s)_{q}^{\alpha}=\frac{1-q^{\alpha}}{1-q}(t-s)_{q}^{\alpha-1}
$$

(IV) The nabla q-derivative of the q-factorial function with respect to $s$ is

$$
\nabla_{q}(t-s)_{q}^{\alpha}=-\frac{1-q^{\alpha}}{1-q}(t-q s)_{q}^{\alpha-1}
$$

For a function $f: \mathbb{T}_{q}^{\alpha} \rightarrow \mathbb{R}$, the left $q$-fractional integral ${ }_{q} \nabla_{a}^{-\alpha}$ of order $\alpha \neq 0,-1,-2, \ldots$ and starting at $0<a \in \mathbb{T}_{q}$ is defined by

$$
{ }_{q} \nabla_{a}^{-\alpha} f(t)=\frac{1}{\Gamma_{q}(\alpha)} \int_{a}^{t}(t-q s)_{q}^{\alpha-1} f(s) \nabla_{q} s
$$

where

$$
\Gamma_{q}(\alpha+1)=\frac{1-q^{\alpha}}{1-q} \Gamma_{q}(\alpha), \quad \Gamma_{q}(1)=1, \alpha>0 .
$$

One should note that the left $q$-fractional integral ${ }_{q} \nabla_{a}^{-\alpha}$ maps functions defined on $\mathbb{T}_{q}$ to functions defined on $\mathbb{T}_{q}^{q}$.

Definition 2 (see [14]). If $0<\alpha \notin \mathbb{N}$, then the Caputo left $q$-fractional derivative of order $\alpha$ of a function $f$ is defined by

$$
\begin{aligned}
{ }_{q} C_{a}^{\alpha} f(t) & :={ }_{q} \nabla_{a}^{-(n-\alpha)} \nabla_{q}^{n} f(t) \\
& =\frac{1}{\Gamma(n-\alpha)} \int_{a}^{t}(t-q s)_{q}^{n-\alpha-1} \nabla_{q}^{n} f(s) \nabla_{q} s,
\end{aligned}
$$

where $n=[\alpha]+1$. In case $\alpha \in \mathbb{N}$, we may write ${ }_{q} C_{a}^{\alpha} f(t):=$ $\nabla_{q}^{n} f(t)$.

Lemma 3 (see [14]). Assume that $\alpha>0$ and $f$ is defined in $a$ suitable domain. Then

$$
{ }_{q} \nabla_{a}^{-\alpha}{ }_{q} C_{a}^{\alpha} f(t)=f(t)-\sum_{k=0}^{n-1} \frac{(t-a)_{q}^{k}}{\Gamma_{q}(k+1)} \nabla_{q}^{k} f(a),
$$

and if $0<\alpha \leq 1$, then

$$
{ }_{q} \nabla_{a}^{-\alpha}{ }_{q} C_{a}^{\alpha} f(t)=f(t)-f(a) .
$$

For solving linear $q$-fractional equations, the following identity is essential:

$$
\begin{array}{r}
{ }_{q} \nabla_{a}^{-\alpha}(x-a)_{q}^{\mu}=\frac{\Gamma_{q}(\mu+1)}{\Gamma_{q}(\alpha+\mu+1)}(x-a)_{q}^{\mu+\alpha}, \\
0<a<x<b,
\end{array}
$$

where $\alpha \in \mathbb{R}^{+}$and $\mu \in(-1, \infty)$. See, for instance, the recent papers $[14,15]$ for more information.

The $q$-analogue of Mittag-Leffler function with double index $(\alpha, \beta)$ is first introduced in [14]. Indeed, it was defined as follows.

Definition 4 (see [14]). For $z, z_{0} \in \mathbf{C}$ and $\mathfrak{R}(\alpha)>0$, the $q$ Mittag-Leffler function is defined by

$$
{ }_{q} E_{\alpha, \beta}\left(\lambda, z-z_{0}\right)=\sum_{k=0}^{\infty} \lambda^{k} \frac{\left(z-z_{0}\right)_{q}^{\alpha k}}{\Gamma_{q}(\alpha k+\beta)} .
$$

In case $\beta=1$, we may use ${ }_{q} E_{\alpha}\left(\lambda, z-z_{0}\right):={ }_{q} E_{\alpha, 1}\left(\lambda, z-z_{0}\right)$.

The following example clarifies how $q$-Mittag-Leffler functions can be used to express the solutions of Caputo $q$ fractional linear initial value problems.

Example 5 (see [14]). Let $0<\alpha \leq 1$ and consider the left Caputo $q$-fractional difference equation

$$
{ }_{q} C_{a}^{\alpha} y(t)=\lambda y(t)+f(t), \quad y(a)=a_{0}, t \in T_{q} .
$$


Applying ${ }_{q} \nabla_{a}^{-\alpha}$ to (16) and using (13), we end up with

$$
y(t)=a_{0}+\lambda_{q} \nabla_{a}^{-\alpha} y(t)+{ }_{q} \nabla_{a}^{-\alpha} f(t) .
$$

To obtain an explicit form for the solution, we apply the method of successive approximation. Set $y_{0}(t)=a_{0}$ and

$$
\begin{array}{r}
y_{m}(t)=a_{0}+\lambda_{q} \nabla_{a}^{-\alpha} y_{m-1}(t)+{ }_{q} \nabla_{a}^{-\alpha} f(t), \\
m=1,2,3, \ldots
\end{array}
$$

For $m=1$, we have by the power formula (14)

$$
y_{1}(t)=a_{0}\left[1+\frac{\lambda(t-a)_{q}^{(\alpha)}}{\Gamma_{q}(\alpha+1)}\right]+{ }_{q} \nabla_{a}^{-\alpha} f(t) .
$$

For $m=2$, we also see that

$$
\begin{aligned}
y_{2}(t)= & a_{0}+\lambda a_{0} \nabla_{a}^{-\alpha}\left[1+\frac{(t-a)_{q}^{\alpha}}{\Gamma_{q}(\alpha+1)}\right] \\
& +{ }_{q} \nabla_{a}^{-\alpha} f(t)+\lambda_{q} \nabla_{a}^{-2 \alpha} f(t) \\
= & a_{0}\left[1+\frac{\lambda(t-a)_{q}^{\alpha}}{\Gamma_{q}(\alpha+1)}+\frac{\lambda^{2}(t-a)_{q}^{2 \alpha}}{\Gamma_{q}(2 \alpha+1)}\right] \\
& +{ }_{q} \nabla_{a}^{-\alpha} f(t)+\lambda_{q} \nabla_{a}^{-2 \alpha} f(t) .
\end{aligned}
$$

If we proceed inductively and let $m \rightarrow \infty$, we obtain the solution

$$
\begin{aligned}
& y(t) \\
& =a_{0}\left[1+\sum_{k=1}^{\infty} \frac{\lambda^{k}(t-a)_{q}^{k \alpha}}{\Gamma_{q}(k \alpha+1)}\right] \\
& +\int_{a}^{t}\left[\sum_{k=1}^{\infty} \frac{\lambda^{k-1}}{\Gamma_{q}(\alpha k)}(t-q s)_{q}^{\alpha k-1}\right] f(s) \nabla_{q} s \\
& =a_{0}\left[1+\sum_{k=1}^{\infty} \frac{\lambda^{k}(t-a)_{q}^{k \alpha}}{\Gamma_{q}(k \alpha+1)}\right] \\
& +\int_{a}^{t}\left[\sum_{k=0}^{\infty} \frac{\lambda^{k}}{\Gamma_{q}(\alpha k+\alpha)}(t-q s)_{q}^{\alpha k+(\alpha-1)}\right] f(s) \nabla_{q} s \\
& =a_{0}\left[1+\sum_{k=1}^{\infty} \frac{\lambda^{k}(t-a)_{q}^{k \alpha}}{\Gamma_{q}(k \alpha+1)}\right] \lambda_{a}^{t}(t-q s)_{q}^{(\alpha-1)}\left[\sum_{k=0}^{\infty} \frac{\lambda^{k}(\alpha k+\alpha)}{\left.\Gamma_{q}\left(t-q^{\alpha} s\right)_{q}^{(\alpha k)}\right] f(s) \nabla_{q} s .}\right. \\
& \left.+\int_{a}^{t}\right]
\end{aligned}
$$

That is,

$$
\begin{aligned}
y(t)= & a_{0 q} E_{\alpha}(\lambda, t-a) \\
& +\int_{a}^{t}(t-q s)_{q}^{\alpha-1}{ }_{q} E_{\alpha, \alpha}\left(\lambda, t-q^{\alpha} s\right) f(s) \nabla_{q} s .
\end{aligned}
$$

Remark 6. If instead we use the modified $q$-Mittag-Leffler function

$$
q^{e_{\alpha, \beta}}\left(\lambda, z-z_{0}\right)=\sum_{k=0}^{\infty} \lambda^{k} \frac{\left(z-z_{0}\right)_{q}^{\alpha k+(\beta-1)}}{\Gamma_{q}(\alpha k+\beta)}
$$

then, the solution representation (17) becomes

$$
y(t)=a_{0} e_{\alpha}(\lambda, t-a)+\int_{a}^{t} q_{\alpha, \alpha}(\lambda, t-q s) f(s) \nabla_{q} s .
$$

Remark 7. If we set $\alpha=1, \lambda=1, a=0$, and $f(t)=0$, we reach to the $q$-exponential formula $e_{q}(t)=\sum_{k=0}^{\infty}\left(t^{k} / \Gamma_{q}(k+1)\right)$ on the time scale $\mathbb{T}_{q}$, where $\Gamma_{q}(k+1)=[k]_{q} !=[1]_{q}[2]_{q} \cdots[k]_{q}$ with $[r]_{q}=\left(1-q^{r}\right) /(1-q)$. It is known that $e_{q}(t)=E_{q}((1-$ $q) t$ ), where $E_{q}(t)$ is a special case of the basic hypergeometric series, given by

$$
E_{q}(t)={ }_{1} \phi_{0}(0 ; q, t)=\prod_{n=0}^{\infty}\left(1-q^{n} t\right)^{-1}=\sum_{n=0}^{\infty} \frac{t^{n}}{(q)_{n}},
$$

where $(q)_{n}=(1-q)\left(1-q^{2}\right) \cdots\left(1-q^{n}\right)$ is the $q$-Pochhammer symbol.

\section{The Main Results}

Throughout the remaining part of the paper, we assume that $0<\alpha \leq 1$. Consider the following $q$-fractional initial value problem:

$$
\begin{aligned}
{ }_{q} C_{a}^{\alpha} y(t) & =f(t, y(t)), \quad a \in \mathbb{T}_{q}, \\
y(a) & =y_{0} .
\end{aligned}
$$

Applying ${ }_{q} \nabla_{a}^{-\alpha}$ to both sides of (26), we obtain

$$
y(t)=y_{0}+{ }_{q} \nabla_{a}^{-\alpha} f(t, y(t)) .
$$

Set

$$
f(t, y(t))=x(t) y(t)
$$

where

$$
0 \leq x(t) \leq \frac{1}{t^{\alpha}(1-q)^{\alpha}}
$$

In the following, we present a comparison result for the fractional summation operator.

Theorem 8. Let $w$ and $v$ satisfy

$$
\begin{aligned}
& w(t) \geq w(a)+{ }_{q} \nabla_{a}^{-\alpha} x(t) w(t), \\
& v(t) \leq v(a)+{ }_{q} \nabla_{a}^{-\alpha} x(t) v(t),
\end{aligned}
$$

respectively. If $w(a) \geq v(a)$, then $w(t) \geq v(t)$ for $t \in \Lambda_{a}=\{a=$ $\left.q^{n_{0}}, q^{n_{0}-1}, \ldots\right\}$. 
Proof. Set $u(t)=v(t)-w(t)$. We claim that $u(t) \leq 0$ for $t \in \Lambda_{a}$. Let us assume that $u(s) \leq 0$ is valid for $s=$ $q^{n_{0}}, q^{n_{0}-1}, \ldots, q^{n-1}$, where $n<n_{0}$. Then, for $t=q^{n}$ we have

$$
\begin{aligned}
u(t) & =v(t)-w(t) \\
& \leq[v(a)-w(a)]+{ }_{q} \nabla_{a}^{-\alpha} x(t)[v(t)-w(t)],
\end{aligned}
$$

or

$$
\begin{aligned}
v(t)-w(t) \leq & {[v(a)-w(a)] } \\
& +\frac{1}{\Gamma_{q}(\alpha)} \int_{a}^{t}(t-q s)_{q}^{\alpha-1} x(s)(v(s)-w(s)) \nabla_{q} s .
\end{aligned}
$$

It follows that

$$
\begin{aligned}
v(t)-w(t) & \\
\leq & {[v(a)-w(a)] } \\
& +\frac{1}{\Gamma_{q}(\alpha)} \int_{a}^{q t}(t-q s)_{q}^{\alpha-1} x(s)(v(s)-w(s)) \nabla_{q} s \\
& +\frac{1}{\Gamma_{q}(\alpha)} \int_{q t}^{t}(t-q s)_{q}^{\alpha-1} x(s)(v(s)-w(s)) \nabla_{q} s .
\end{aligned}
$$

Since $v(t)-w(t) \leq 0$ and $\int_{\rho(t)}^{t} f(s) \nabla s=(t-\rho(t)) f(t),(34)$ can be written in the form

$$
\begin{aligned}
v(t) & -w(t) \\
& \leq \frac{1}{\Gamma_{q}(\alpha)}(t-q t)(t-q t)_{q}^{\alpha-1} x(t)(v(t)-w(t)) \\
& =(1-q)^{\alpha} t^{\alpha} x(t)(v(t)-w(t)),
\end{aligned}
$$

where $\Gamma_{q}(\alpha)=(1-q)_{q}^{\alpha-1} /(1-q)^{\alpha-1}$ is used. It follows that

$$
\left(1-x(t)(1-q)^{\alpha} t^{\alpha}\right)(v(t)-w(t)) \leq 0 .
$$

By (29), we conclude that $v(t)-w(t) \leq 0$.

Define the following operator

$$
{ }_{q} \Omega_{x} \phi={ }_{q} \nabla_{a}^{-\alpha} x(t) \phi(t) .
$$

The following lemmas are essential in the proof of the main theorem. We only state these statements as their proofs are straightforward.

Lemma 9. For any constant $\lambda$, one has

$$
\left|{ }_{q} \Omega_{\lambda} 1\right| \leq{ }_{q} \Omega_{|\lambda|} 1 .
$$

Lemma 10. For any constant $\lambda$, one has

$$
{ }_{q} \Omega_{\lambda}^{n} 1=\frac{\lambda^{n}(t-a)_{q}^{n \alpha}}{\Gamma_{q}(n \alpha+1)}, \quad \text { where } n \in \mathbb{N} .
$$

Lemma 11. Let $\lambda>0$ be such that $|y(t)| \leq \lambda$ for $t \in \Lambda_{a}$. Then

$$
\left|{ }_{q} \Omega_{y}^{n} 1\right| \leq{ }_{q} \Omega_{\lambda}^{n} 1, \quad n \in \mathbb{N} .
$$

The next result together with Theorem 8 will give us the desired $q$-fractional Gronwall-type inequality.

Theorem 12. Let $|x(t)| \leq 1 /(1-q)^{\alpha} t^{\alpha}$ for $t \in \Lambda_{a} \cap[a, b]$. Then, the q-fractional integral equation

$$
y(t)=y(a)+{ }_{q} \nabla_{a}^{-\alpha} x(t) y(t),
$$

for $t \in \Lambda_{a} \cap[a, b]$ where $b \in \mathbb{R}$, has a solution

$$
y(t)=y(a) \sum_{k=0}^{\infty} \Omega_{x}^{k} 1 .
$$

Proof. The proof is achieved by utilizing the successive approximation method. Set

$$
\begin{gathered}
y_{0}(t)=y(a), \\
y_{n}(t)=y(a)+{ }_{q} \nabla_{a}^{-\alpha} x(t) y_{n-1}(t), \quad \text { for } n \geq 1 .
\end{gathered}
$$

We observe that

$$
\begin{aligned}
y_{1}(t) & =y(a)+{ }_{q} \nabla_{a}^{-\alpha} x(t) y_{0}(t) \\
& =y(a)+{ }_{q} \Omega_{x} y(a), \\
y_{2}(t) & =y(a)+{ }_{q} \Omega_{x}\left(y(a)+{ }_{q} \Omega_{x} y(a)\right) \\
& =y(a)+{ }_{q} \Omega_{x} y(a)+{ }_{q} \Omega_{x}^{2} y(a) .
\end{aligned}
$$

Inductively, we end up with

$$
y_{n}(t)=y(a) \sum_{k=0}^{n} \Omega_{x}^{k} 1, \quad n \geq 0 .
$$

Taking the limit as $n \rightarrow \infty$, we have

$$
y(t)=y(a) \sum_{k=0}^{\infty} \Omega_{x}^{k} 1 .
$$

It remains to prove the convergence of the series in (46). The subsequent analyses are carried out for $a=0$.

In virtue of (29), we obtain

$$
\begin{aligned}
\sum_{k=0}^{\infty} q_{q} \Omega_{x}^{k} 1 & \leq \sum_{k=0}^{\infty} \Omega_{1 / t^{\alpha}(1-q)^{\alpha}}^{k} \\
& \leq \sum_{k=0}^{\infty}\left(q_{q} \nabla_{0}^{-\alpha}\right)^{k}\left(\frac{1}{t^{\alpha}(1-q)^{\alpha}}\right) \\
& \leq \frac{1}{(1-q)^{\alpha}} \sum_{k=0}^{\infty}\left(q_{q} \nabla_{0}^{-\alpha}\right)^{k}\left(t^{-\alpha}\right) .
\end{aligned}
$$

However, for $k=1$ we observe that

$$
{ }_{q} \nabla_{0}^{-\alpha} t^{-\alpha}=\frac{\Gamma(1-\alpha)}{\Gamma(0+1)} t^{0}=\Gamma_{q}(1-\alpha) .
$$


For $k=2$, it follows that

$$
\begin{aligned}
{ }_{q} \nabla_{0}^{-\alpha} & \left(\Gamma_{q}(1-\alpha)\right) \\
& =\Gamma_{q}(1-\alpha)_{q} \nabla_{0}^{-\alpha} t^{0}=\frac{\Gamma_{q}(1-\alpha)}{\Gamma_{q}(\alpha+1)} t^{\alpha}
\end{aligned}
$$

For $k=3$, we have

$$
{ }_{q} \nabla_{0}^{-\alpha}\left(\frac{\Gamma_{q}(1-\alpha)}{\Gamma_{q}(\alpha+1)} t^{\alpha}\right)=\frac{\Gamma_{q}(1-\alpha)}{\Gamma_{q}(\alpha+1)} \frac{\Gamma_{q}(\alpha+1)}{\Gamma_{q}(\alpha+\alpha+1)} t^{2 \alpha},
$$

or

$$
{ }_{q} \nabla_{0}^{-\alpha}\left(\frac{\Gamma_{q}(1-\alpha)}{\Gamma_{q}(\alpha+1)} t^{\alpha}\right)=\frac{\Gamma_{q}(1-\alpha)}{\Gamma_{q}(2 \alpha+1)} t^{2 \alpha}
$$

For $k=4$, we get

$$
{ }_{q} \nabla_{0}^{-\alpha}\left(\frac{\Gamma_{q}(1-\alpha)}{\Gamma_{q}(2 \alpha+1)} t^{2 \alpha}\right)=\frac{\Gamma_{q}(1-\alpha)}{\Gamma_{q}(3 \alpha+1)} t^{3 \alpha} .
$$

Therefore, (47) becomes

$$
\begin{aligned}
& \sum_{k=0}^{\infty} q^{k} \Omega_{x}^{k} 1 \\
& \leq \frac{1}{(1-q)^{\alpha}}\left[1+\Gamma_{q}(1-\alpha)+\Gamma_{q}(1-\alpha) \sum_{k=1}^{\infty} \frac{t^{k \alpha}}{\Gamma_{q}(k \alpha+1)}\right] .
\end{aligned}
$$

Let $a_{k}=t^{(k-1) \alpha} / \Gamma_{q}((k-1) \alpha+1)$. Then,

$$
\begin{aligned}
\frac{a_{k}}{a_{k-1}} & =\frac{t^{k \alpha}}{\Gamma_{q}(k \alpha+1)} \frac{\Gamma_{q}((k-1) \alpha+1)}{t^{(k-1) \alpha}} \\
& =t^{\alpha} \frac{\Gamma_{q}((k-1) \alpha+1)}{\Gamma_{q}(k \alpha+1)} \leq \frac{\Gamma_{q}((k-1) \alpha+1)}{\Gamma_{q}(k \alpha+1)} .
\end{aligned}
$$

We observe that

$$
\begin{aligned}
& \frac{\Gamma_{q}((k-1) \alpha+1)}{\Gamma_{q}(k \alpha+1)} \\
& \quad=\frac{(1-q)_{q}^{(k-1) \alpha}(1-q)^{(1-k) \alpha}}{(1-q)_{q}^{k \alpha}(1-q)^{-k \alpha}}=\frac{(1-q)_{q}^{(k-1) \alpha}(1-q)^{\alpha}}{(1-q)_{q}^{k \alpha}} .
\end{aligned}
$$

Setting

$$
\begin{aligned}
& \frac{(1-q)_{q}^{(k-1) \alpha}(1-q)^{\alpha}}{(1-q)_{q}^{k \alpha}} \\
& :=(1-q)^{\alpha} \frac{\prod_{i=0}^{\infty}\left(\left(1-q^{i+1}\right) /\left(1-q^{i} q^{k \alpha-\alpha+1}\right)\right)}{\prod_{i=0}^{\infty}\left(\left(1-q^{i+1}\right) /\left(1-q^{i} q^{k \alpha+1}\right)\right)},
\end{aligned}
$$

we deduce that

$$
\begin{gathered}
\lim _{k \rightarrow \infty}(1-q)^{\alpha} \frac{\prod_{i=0}^{\infty}\left(\left(1-q^{i+1}\right) /\left(1-q^{i} q^{k \alpha-\alpha+1}\right)\right)}{\prod_{i=0}^{\infty}\left(\left(1-q^{i+1}\right) /\left(1-q^{i} q^{k \alpha+1}\right)\right)} \\
=(1-q)^{\alpha} \frac{\prod_{i=0}^{\infty}\left(1-q^{i+1}\right)}{\prod_{i=0}^{\infty}\left(1-q^{i+1}\right)}=(1-q)^{\alpha}<1 .
\end{gathered}
$$

Hence, convergence is guaranteed. In case $a>0$, we can proceed in a similar way taking into account that $(t-a)_{q}^{k \alpha} /(t-$ $a)_{q}^{k \alpha-\alpha}=\left(t-q^{k \alpha} q^{\alpha} a\right)_{q}^{\alpha} \rightarrow t^{\alpha}$ as $k \rightarrow \infty$.

Theorem 13 ( $q$-fractional Gronwall's lemma). Let $v$ and $\mu$ be nonnegative real valued functions such that $0 \leq \mu(t)<1 / t^{\alpha}(1-$ $q)^{\alpha}$ for all $t \in \Lambda_{a}$ (in particular if $\left.0 \leq \mu(t)<1 /(1-q)^{\alpha}\right)$ and

$$
v(t) \leq v(a)+{ }_{q} \nabla_{a}^{-\alpha} v(t) \mu(t) .
$$

Then

$$
v(t) \leq v(a) \sum_{k=0}^{\infty} \Omega_{\mu}^{k} 1 .
$$

The proof of the previous statement is a straightforward implementation of Theorems 8 and 12 by setting $w(t)=$ $v(a) \sum_{k=0}^{\infty}\left(\Omega_{\mu}^{k} 1\right)(t)$.

In case $\alpha=1$, we deduce the following immediate consequence of Theorem 13 which can be considered as the well-known $q$-Gronwall's Lemma; consult, for instance, the paper [42].

Corollary 14. Let $0 \leq \delta(t)<1 /(1-q)$ for all $t \in \Lambda_{a}$. If

$$
v(t) \leq v(a)+\int_{a}^{t} \delta(s) v(s) \nabla_{q} s,
$$

then

$$
v(t) \leq v(a) e_{q}(t, a),
$$

where $e_{q}(t, a)={ }_{q} \Omega_{1}(1, t-a)$ is the nabla q-exponential function on the time scale $\mathbb{T}_{q}$.

\section{Applications}

In this section, we show, by the help of the $q$-fractional Gronwall inequality proved in the previous section, that small changes in the initial conditions of Caputo $q$-fractional initial value problems lead to small changes in the solution.

Let $f(t, y)$ satisfy a Lipschitz condition with constant $0 \leq$ $L<1$ for all $t$ and $y$.

Example 15. Consider the following $q$-fractional initial value problems:

$$
\begin{gathered}
{ }_{q} \nabla_{a}^{\alpha} \varphi(t)=f(t, \varphi(t)), \quad 0<\alpha \leq 1, a \in \mathbb{T}_{q}, t \in \Lambda_{a}, \\
\varphi(a)=\gamma, \\
{ }_{q} \nabla_{a}^{\alpha} \psi(t)=f(t, \psi(t)), \quad 0<\alpha \leq 1, a \in \mathbb{T}_{q}, t \in \Lambda_{a}, \\
\psi(a)=\beta .
\end{gathered}
$$


It follows that

$$
\varphi(t)-\psi(t)=(\gamma-\beta)+{ }_{q} \nabla_{a}^{-\alpha}[f(t, \varphi(t))-f(t, \psi(t))] .
$$

Taking the absolute value, we obtain

$$
|\varphi(t)-\psi(t)| \leq|\gamma-\beta|+\left|{ }_{q} \nabla_{a}^{-\alpha} f(t, \varphi(t))-f(t, \psi(t))\right|,
$$

or

$$
|\varphi(t)-\psi(t)| \leq|\gamma-\beta|+L_{q} \nabla_{a}^{-\alpha}|\varphi(t)-\psi(t)| \text {. }
$$

By using Theorem 13, we get

$$
|\varphi(t)-\psi(t)| \leq|\gamma-\beta| \sum_{i=0}^{\infty} q \Omega_{L}^{i} 1=|\gamma-\beta|{ }_{q} \Omega_{\alpha}(L, t-a) .
$$

Consider the following $q$-fractional initial value problem:

$$
\begin{gathered}
{ }_{q} \nabla_{a}^{\alpha} \phi(t)=f(t, \phi(t)), \quad 0<\alpha \leq 1, a \in \mathbb{T}_{q}, t \in \Lambda_{a}, \\
\phi(a)=\gamma_{n},
\end{gathered}
$$

where $\gamma_{n} \rightarrow \gamma$. If the solution of (67) is denoted by $\phi_{n}$, then for all $t \in \Lambda_{a}$ we have

$$
\left|\varphi(t)-\phi_{n}(t)\right| \leq\left|\gamma-\gamma_{n}\right| \sum_{i=0}^{\infty} q^{i} \Omega_{L}^{i} 1=\left|\gamma-\gamma_{n}\right|_{q} \Omega_{\alpha}(L, t-a) .
$$

Hence $\left|\varphi(t)-\phi_{n}(t)\right| \rightarrow 0$ as $\gamma_{n} \rightarrow \gamma$. This clearly verifies the dependence of solutions on the initial conditions.

\section{References}

[1] S. G. Samko, A. A. Kilbas, and O. I. Marichev, Fractional Integrals and Derivatives: Theory and Applications, Gordon and Breach Science, Yverdon, Switzerland, 1993.

[2] I. Podlubny, Fractional Differential Equations, vol. 198, Academic Press, San Diego, Calif, USA, 1999.

[3] A. A. Kilbas, H. M. Srivastava, and J. J. Trujillo, Theory and Applications of Fractional Differential Equations, vol. 204 of North-Holland Mathematics Studies, Elsevier Science B.V., Amsterdam, The Netherlands, 2006.

[4] R. Finkelstein and E. Marcus, "Transformation theory of the $q$ oscillator," Journal of Mathematical Physics, vol. 36, no. 6, pp. 2652-2672, 1995.

[5] R. J. Finkelstein, “The q-Coulomb problem," Journal of Mathematical Physics, vol. 37, no. 6, pp. 2628-2636, 1996.

[6] R. Floreanini and L. Vinet, "Automorphisms of the $q$-oscillator algebra and basic orthogonal polynomials," Physics Letters A, vol. 180, no. 6, pp. 393-401, 1993.

[7] R. Floreanini and L. Vinet, "Symmetries of the $q$-difference heat equation," Letters in Mathematical Physics, vol. 32, no. 1, pp. 3744, 1994.

[8] R. Floreanini and L. Vinet, "Quantum symmetries of $q$ difference equations," Journal of Mathematical Physics, vol. 36, no. 6, pp. 3134-3156, 1995.
[9] P. G. O. Freund and A. V. Zabrodin, "The spectral problem for the $q$-Knizhnik-Zamolodchikov equation and continuous $q$ Jacobi polynomials," Communications in Mathematical Physics, vol. 173, no. 1, pp. 17-42, 1995.

[10] G.-N. Han and J. Zeng, "On a q-sequence that generalizes the median Genocchi numbers," Annales des Sciences Mathématiques du Québec, vol. 23, no. 1, pp. 63-72, 1999.

[11] J. O. Alzabut and T. Abdeljawad, "Perron's theorem for $q$ delay difference equations," Applied Mathematics \& Information Sciences, vol. 5, no. 1, pp. 74-84, 2011.

[12] V. Kac and P. Cheung, Quantum Calculus, Springer, New York, NY, USA, 2002.

[13] F. H. Jackson, " $q$-difference equations," American Journal of Mathematics, vol. 32, no. 4, pp. 305-314, 1910.

[14] T. Abdeljawad and D. Baleanu, "Caputo $q$-fractional initial value problems and a $q$-analogue Mittag-Leffler function," Communications in Nonlinear Science and Numerical Simulation, vol. 16, no. 12, pp. 4682-4688, 2011.

[15] T. Abdeljawad and D. Baleanu, "Fractional differences and integration by parts," Journal of Computational Analysis and Applications, vol. 13, no. 3, pp. 574-582, 2011.

[16] T. Abdeljawad, B. Benli, and D. Baleanu, "A generalized $q$ Mittag-Leffler function by $q$-Captuo fractional linear equations," Abstract and Applied Analysis, vol. 2012, Article ID 546062, 11 pages, 2012.

[17] F. Jarad, T. Abdeljawad, and D. Baleanu, "Stability of $q$-fractional non-autonomous systems," Nonlinear Analysis: Real World Applications, vol. 14, no. 1, pp. 780-784, 2013.

[18] T. Abdeljawad, F. Jarad, and D. Baleanu, "A semigroup-like property for discrete Mittag-Leffler functions," Advances in Difference Equations, vol. 2012, article 72, 2012.

[19] N. R. O. Bastos, R. A. C. Ferreira, and D. F. M. Torres, "Necessary optimality conditions for fractional difference problems of the calculus of variations," Discrete and Continuous Dynamical Systems A, vol. 29, no. 2, pp. 417-437, 2011.

[20] N. R. O. Bastos, R. A. C. Ferreira, and D. F. M. Torres, "Discretetime variational problems," Signal Process, vol. 91, no. 3, 2011.

[21] F. M. Atıcı and S. Şengül, "Modeling with fractional difference equations," Journal of Mathematical Analysis and Applications, vol. 369, no. 1, pp. 1-9, 2010.

[22] F. M. Atici and P. W. Eloe, "A transform method in discrete fractional calculus," International Journal of Difference Equations, vol. 2, no. 2, pp. 165-176, 2007.

[23] F. M. Atici and P. W. Eloe, "Initial value problems in discrete fractional calculus," Proceedings of the American Mathematical Society, vol. 137, no. 3, pp. 981-989, 2009.

[24] C. S. Goodrich, "Continuity of solutions to discrete fractional initial value problems," Computers \& Mathematics with Applications, vol. 59, no. 11, pp. 3489-3499, 2010.

[25] C. S. Goodrich, "Solutions to a discrete right-focal fractional boundary value problem," International Journal of Difference Equations, vol. 5, no. 2, pp. 195-216, 2010.

[26] G. A. Anastassiou, "Nabla discrete fractional calculus and nabla inequalities," Mathematical and Computer Modelling, vol. 51, no. 5-6, pp. 562-571, 2010.

[27] J.-F. Cheng and Y.-M. Chu, "Fractional difference equations with real variable," Abstract and Applied Analysis, vol. 2012, Article ID 918529, 24 pages, 2012.

[28] J. F. Cheng and G. C. Wu, "Solutions of fractional difference equations of order $(2, q)$," Acta Mathematica Sinica, vol. 55, no. 3, pp. 469-480, 2012. 
[29] W. A. Al-Salam, "Some fractional q-integrals and q-derivatives," Proceedings of the Edinburgh Mathematical Society II, vol. 15, no. 2, pp. 135-140, 1966.

[30] R. P. Agarwal, "Certain fractional q-integrals and q-derivatives," Proceedings of the Cambridge Philosophical Society, vol. 66, pp. 365-370, 1969.

[31] W. A. Al-Salam and A. Verma, "A fractional Leibniz q-formula," Pacific Journal of Mathematics, vol. 60, no. 2, pp. 1-9, 1975.

[32] F. M. Atici and P. W. Eloe, "Fractional q-calculus on a time scale," Journal of Nonlinear Mathematical Physics, vol. 14, no. 3, pp. 333-344, 2007.

[33] P. M. Rajković, S. D. Marinković, and M. S. Stanković, "Fractional integrals and derivatives in q-calculus," Applicable Analysis and Discrete Mathematics, vol. 1, no. 1, pp. 311-323, 2007.

[34] Z. S. I. Mansour, "Linear sequential $q$-difference equations of fractional order," Fractional Calculus \& Applied Analysis, vol. 12, no. 2, pp. 159-178, 2009.

[35] Y. Zhao, H. Chen, and Q. Zhang, "Existence results for fractional $q$-difference equations with nonlocal $q$-integral boundary conditions," Advances in Difference Equations, vol. 2013, article 48, 2013.

[36] M. H. Annaby and Z. S. Mansour, q-fractional Calculus and Equations, vol. 2056 of Lecture Notes in Mathematics, Springer, Heidelberg, Germany, 2012.

[37] H. Ye, J. Gao, and Y. Ding, "A generalized Gronwall inequality and its application to a fractional differential equation," Journal of Mathematical Analysis and Applications, vol. 328, no. 2, pp. 1075-1081, 2007.

[38] Q.-H. Ma and J. Pečarić, "Some new explicit bounds for weakly singular integral inequalities with applications to fractional differential and integral equations," Journal of Mathematical Analysis and Applications, vol. 341, no. 2, pp. 894-905, 2008.

[39] K. M. Furati and N.-E. Tatar, "Inequalities for fractional differential equations," Mathematical Inequalities \& Applications, vol. 12, no. 2, pp. 279-293, 2009.

[40] Q.-X. Kong and X.-L. Ding, "A new fractional integral inequality with singularity and its application," Abstract and Applied Analysis, vol. 2012, Article ID 937908, 12 pages, 2012.

[41] F. M. Atıc1 and P. W. Eloe, "Gronwall's inequality on discrete fractional calculus," Computers \& Mathematics with Applications, vol. 64, no. 10, pp. 3193-3200, 2012.

[42] W. N. Li and W. Sheng, "Some Gronwall type inequalities on time scales," Journal of Mathematical Inequalities, vol. 4, no. 1, pp. 67-76, 2010. 


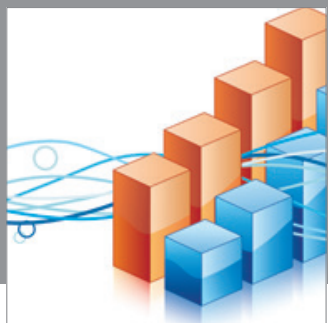

Advances in

Operations Research

mansans

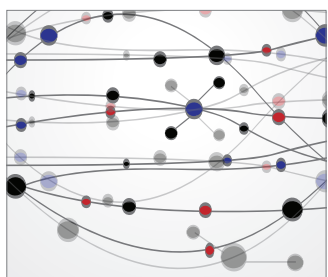

The Scientific World Journal
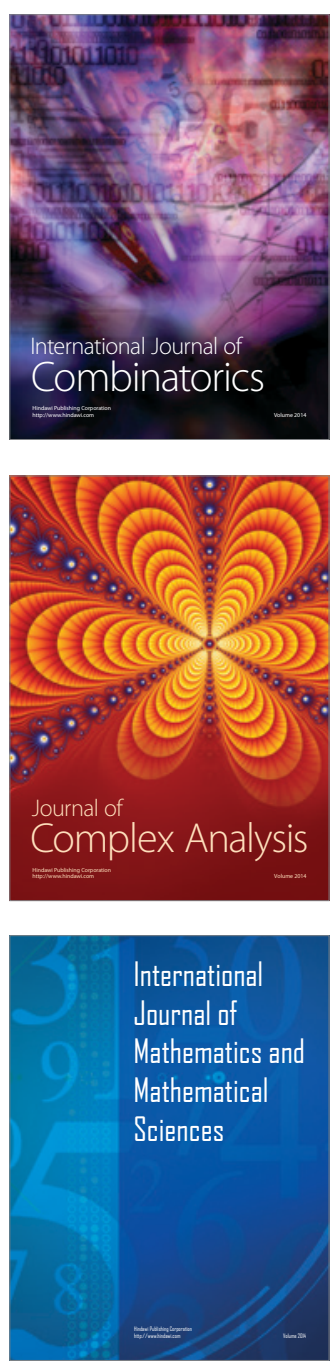
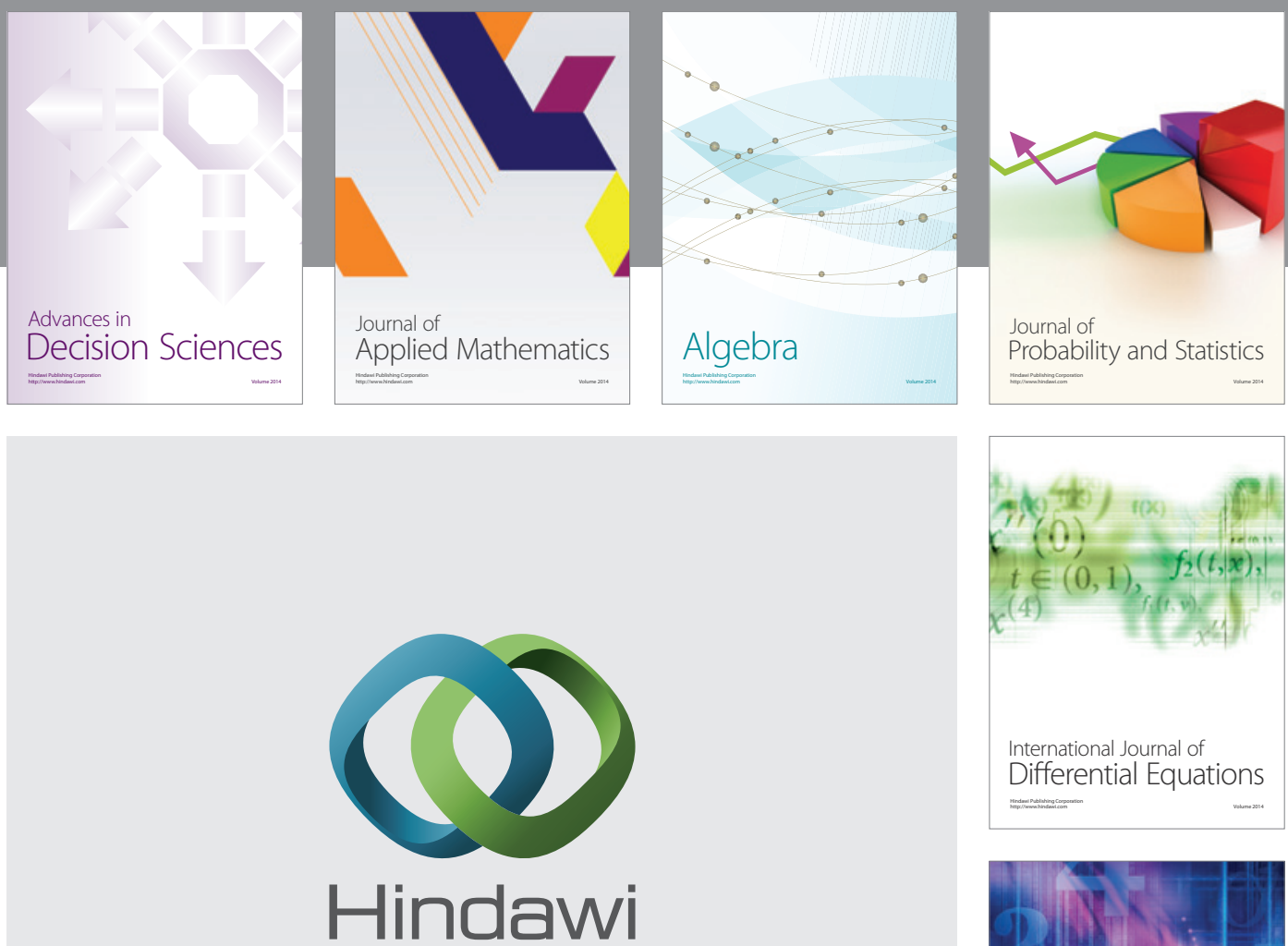

Submit your manuscripts at http://www.hindawi.com
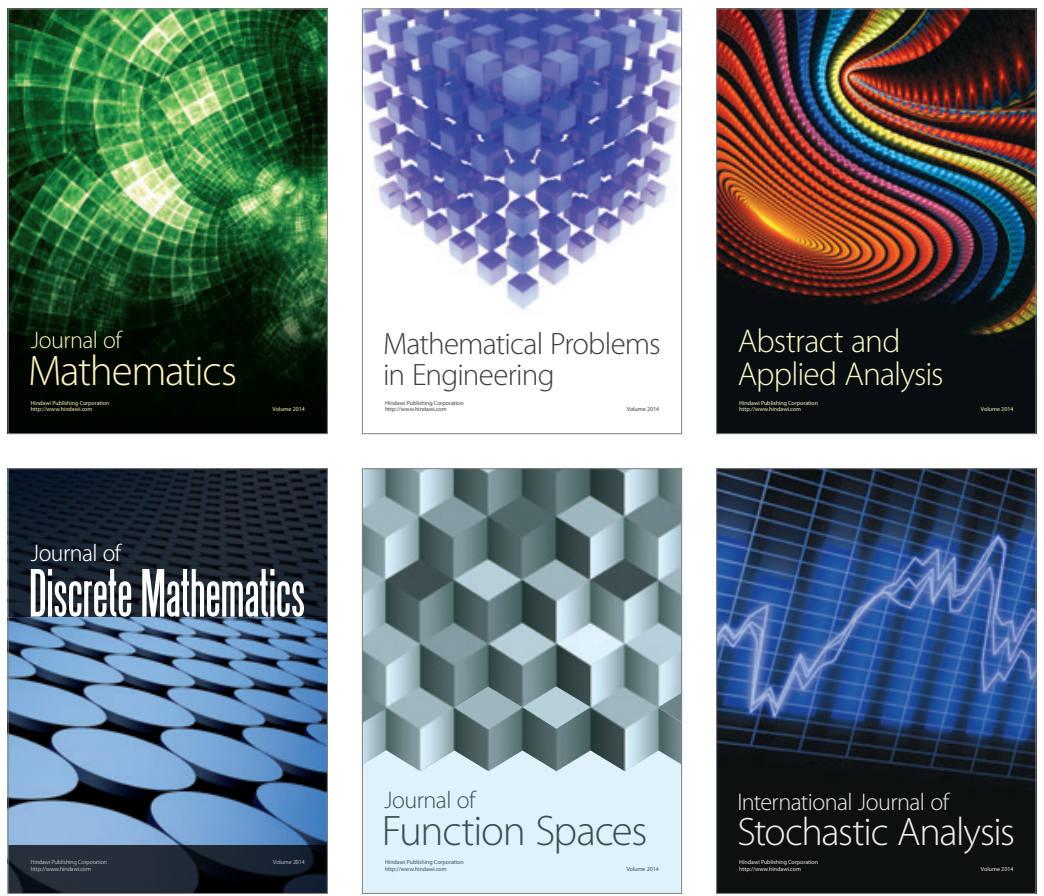

Journal of

Function Spaces

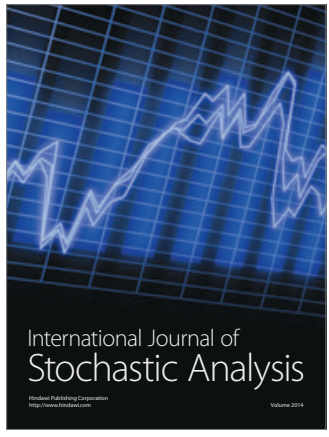

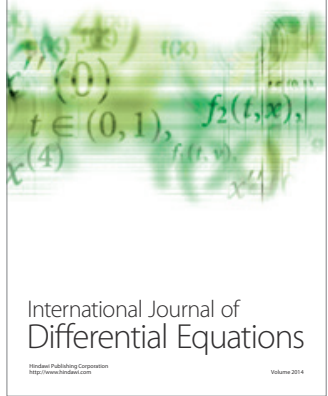
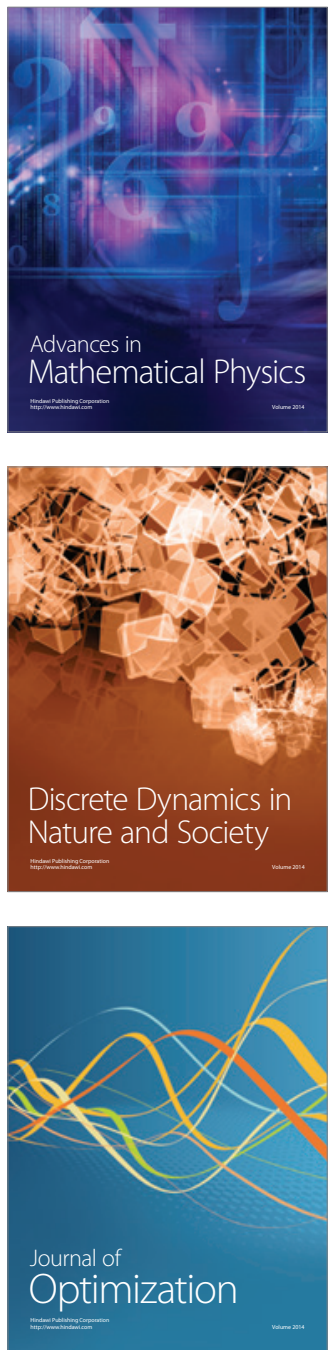